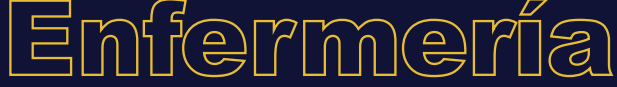 \\ .
}

\section{Relación entre estrategias de aprendizaje y rendimiento académico: estudiantes de enfermería, Universidad Santo Tomás}

\section{Relationship between learning strategies and academic performance: nursing students, University Santo Tomas}

\section{Relação entre estratégias de aprendizagem e desempenho escolar: estudantes de enfermagem, Universidade Santo Tomás}

C. Mercado-Elgueta ${ }^{\mathrm{a} 1}{ }^{*}$, M. Illesca-Pretty ${ }^{\mathrm{b} 2}$, A. Hernández-Díaz ${ }^{\mathrm{c} 3 *}$

ORCID:

a 0000-0001-5060-4513

b 0000-0003-0635-5331

c $0000-0003-3380-2846$

${ }^{1}$ Carrera de Enfermería, Universidad Santo Tomas, Temuco, Chile

${ }^{2}$ Departamento Medicina Interna, Oficina de Educación en Ciencias de la Salud, Facultad de Medicina Universidad de La Frontera, Temuco, Chile

${ }^{3}$ Escuela de Enfermería, Facultad de Ciencias, Universidad Mayor, Chile

Recibido: 5 marzo 2018

Aceptado: 15 octubre 2018

\section{Resumen}

Las estrategias de aprendizaje son actividades o procesos mentales que llevan a cabo los/as estudiantes, intencionalmente para procesar, entender y adoptar la información que reciben en el proceso educativo. Objetivo: Describir las estrategias de aprendizaje de estudiantes de la Carrera de Enfermería, Universidad Santo Tomás Temuco-Chile, y determinar la correspondencia entre ellas con el rendimiento académico y variables sociodemográficas.

Métodos: Se realizó un estudio no experimental de corte transversal. La muestra, no-probabilística, de conveniencia, conformada por 185 estudiantes de enfermería de todos los niveles. Se utilizó la Escala de Estrategias de Aprendizaje ACRA-Abreviada (Adquisición, Codificación, Recuperación y Apoyo de la 
información). Se usó el Programa Stata 11.0, para comparar promedios la prueba t-test y ANOVA, y la correlación de Pearson, para la relación entre variables cuantitativas.

Resultados: Los estudiantes emplean un número importante de estrategias, principalmente las de repetición y relectura (adquisición) y las de apoyo social (apoyo). Las mujeres utilizan más estrategias que los hombres, encontrándose diferencias en las de selección y organización (codificación) de elaboración (codificación) y de subrayado (adquisición). No se encontraron relaciones consistentes entre el uso de estrategias y el rendimiento académico.

Conclusión: Se conocen un número importante de estrategias y hacen uso de ellas, pero no de manera profunda, empleándolas básicamente como elementos de memorización y apoyo al aprendizaje. Se propone, realizar diagnóstico de estrategias al ingreso universitario, capacitación docente y establecer programas donde se promueva la capacidad en los educandos de gestionar su aprendizaje, y la evaluación autentica de los contenidos.

Palabras clave: Estudiantes de enfermería; educación en enfermería; evaluación de programa de enfermería; Chile.

\section{Abstract}

Learning strategies are mental processes or activities intentionally carried out by students to process, understand, and adopt the information being received during the education process.

Objective: To describe the learning strategies of students in the Nursing Program, Santo Tomas University, Temuco-Chile, and determine their relationships with academic performance and other sociodemographic variables.

Methods: This is a transversal and non-experimental study with a non-probabilistic and by convenience sample constituted by 185 nursing students from all levels. The ACRS-Abbreviated Learning Strategies Scale (Acquisition, Coding, Recall, Support to information) was used. The Strata 11.0 was used to calculate averages, T-test, ANOVA, and Pearson correlations.

Results: Students use diverse strategies, mainly those related to repetition and rereading (acquisition) and those related to social support. Female students use more strategies than male students; and differences were found on those strategies related to selection, organization and elaboration (coding), and highlighting (acquisition). No consistent relationships between the use of strategies and the academic performance were found.

Conclusion: While several learning strategies are known, though not extensively used, they are basically applied as a memorization tool. A proposal is presented to develop the assessment of learning strategies during enrollment, and support teacher training, while at the same time establishing programs to improve the capacity of students to manage their learning process.

Keywords: Students, nursing; education, nursing; nursing evaluation research; Chile.

\section{Resumo}

As estratégias de aprendizagem são atividades ou processos mentais que realizam os/as estudantes, intencionalmente para processar, entender e adoptar a informação que recebem no processo educativo. Objetivo: Descrever as estratégias de aprendizagem de estudantes da Carreira de Enfermagem, Universidade Santo Tomás Temuco-Chile, e determinar a correspondência entre elas com o desempenho escolar e variáveis sociodemográficas.

Métodos: Realizou-se um estudo não experimental de corte transversal. A amostragem, não-probabilística, de conveniência, conformada por 185 estudantes de enfermagem de todos os níveis. Utilizou-se a Escala de Estratégias de Aprendizagem ACRA-Abreviada (Aquisição, Codificação, Recuperação e Apoio da informação). Usou-se o Programa Stata 11.0, para comparar a média da proba t-test e Anova, e a correlação de Pearson, para a relação entre variáveis quantitativas. 
Resultados: Os estudantes empregam um número importante de estratégias, principalmente as de repetição e releitura (aquisição) e as de apoio social (apoio). As mulheres utilizam mais estratégias quanto os homens, encontrando-se diferenças nas de seleção e organização (codificação) de elaboração (codificação) e de sublinhado (aquisição). Não se encontraram relações consistentes entre o uso de estratégias e o desempenho escolar.

Conclusão: Conhecem-se um número importante de estratégias e fazem uso delas, mas não de maneira profunda, empregando-as basicamente como elementos de memorização e apoio à aprendizagem. Propõe-se, realizar diagnóstico de estratégias para o ingresso universitário, capacitação docente e estabelecer programas onde se promova a capacidade nos aprendizes de gerenciar sua aprendizagem, e a avaliação autentica dos conteúdos.

Palavras chave: Estudantes de enfermagem; educação em enfermagem; avaliação de programa de enfermagem; Chile.

\section{Introducción}

Una nueva mirada en la educación implica no sólo un cambio profundo de tipo estructural, centrado en la adecuación de las Universidades a determinados rasgos formales comunes a todas las Instituciones de Educación Superior ${ }^{1}$, sino, un enfoque diferente de la docencia, en la cual se produzcan cambios que incidan de manera positiva en el binomio enseñanza-aprendizaje².

En este contexto, un factor clave en la innovación docente universitaria radica en desplazar su punto de gravedad desde el énfasis en la enseñanza hacia la prioridad del aprendizaje. De este modo, la principal función del docente es facilitar y guiar al estudiante para que pueda acceder intelectualmente a los contenidos y prácticas profesionales de una determinada disciplina ${ }^{3}$. Esto requiere de un sistema de aprendizaje autónomo y tutorizado, que posibilitará al educando llegar a construir el conocimiento e interpretar de forma significativa el mundo que le rodea ${ }^{4}$, por lo cual es imprescindible considerar que el aprendizaje ha de concebirse como un proceso que tiene lugar a lo largo de toda la vida ${ }^{5}$.

Esta concepción debe descansar en el desarrollo de estrategias fundamentadas en principios de tipo constructivista que permitan al estudiante aprender a aprender, lo cual genere un ambiente que les propicie incrementar su autonomía y fomente el pensamiento crítico y la reflexión sobre su proceso de aprendi$z^{2} \mathrm{je}^{6}$. Así mismo, el profesorado debe emplear didácticas educativas que faciliten aprender a hacer y aprender de forma colaborativa junto a sus iguales ${ }^{7}$. Puesto que el principal protagonista en la Educación Superior es el alumnado, implica atender a los componentes cognitivos y afectivo-motivacionales del aprendizaje. Respecto a los primeros, se plantea que las llamadas estrategias cognitivas o de aprendizaje integran pensamientos y comportamientos, que facilitarían la adquisición de información y su integración con los conocimientos previos ya existentes, así como la recuperación de la información disponible ${ }^{8}$. A su vez, existe una clasificación de estrategias en adquisición de información, apoyo al proceso de información y codificación de información?

El aprendizaje estratégico centra su atención en los pensamientos, comportamientos, motivaciones, actitudes y creencias, tanto encubiertas como manifiestas, que se relacionan significativamente con el éxito en el aprendizaje a nivel universitario y que pueden ser alterados con intervenciones educativas, como lo demuestran en diversas investigaciones ${ }^{10}$.

En concordancia con lo anterior, es evidente la necesidad de analizar y desarrollar las estrategias de aprendizaje de los estudiantes universitarios ${ }^{11}$; así como de diseñar e implementar en la universidad programas para acrecentar la motivación de los educandos y la autorregulación durante el estudio, lo cual mejora la toma de conciencia y control sobre lo que va a aprender, cómo va a aprender e incrementar la calidad de su educación y el rendimiento académico ${ }^{12}$.

El estudio de la relación existente entre el rendimiento académico y las estrategias de aprendizaje ha ido aumentando considerablemente en los últimos años ${ }^{13-15}$. Este interés viene determinado, 
fundamentalmente, por dos aspectos. En primer lugar, la aparición de trabajos que derriban la idea, ampliamente extendida en las comunidades universitarias, de que un estudiante en este nivel, por el simple hecho de serlo, se encuentra perfectamente capacitado para afrontar sus estudios con éxito, que éste depende exclusivamente de su esfuerzo ${ }^{12,16,17}$. En segundo lugar, debido a los cambios propuestos por el Espacio Europeo de Educación Superior, el cual genera una nueva estructura, en la que el peso del proceso de enseñanza-aprendizaje pasa de la labor docente del profesor, a la actividad que desarrolla el estudiante, dado que se comienza a reconocer el trabajo que este realiza de manera individual o en grupo, con asesoría en tutorías, y disminución en número de clases presenciales, lo que aumenta la importancia de la autonomía y la capacidad de autorregulación a la hora de afrontar sus estudios ${ }^{12}$.

Chile no ha quedado ajeno a estas innovaciones educativas. Desde 1981, se ha producido un cambio paradigmático con la creación de Universidades Privadas, Institutos Profesionales y Centros de Formación Técnica, lo que provocó aumento en el porcentaje de matrículas, que había caído un 30\% desde 1975, y diversificación del sistema de Educación Superior al estimular la competencia entre las Instituciones ${ }^{18}$. Todo ello, conllevó a una masificación del acceso, lo cual implicó una población estudiantil más grande y diversa ${ }^{19}$, esto trajo como consecuencia, que en muchas ocasiones ingresan alumnos a la Educación Superior sin las competencias mínimas que requieren los Planes de Estudio ${ }^{13}$, más aún, la mayoría no son capaces de autorregular su propio proceso de aprendizaje. Desde hace tiempo, se advirtió que la falta de estrategias y procesos de autorregulación para enfrentar el proceso de formación es el factor principal del fracaso universitario ${ }^{20}$. Este planteamiento genera dos situaciones posibles: persistencia en sus ideales a costa de muchos años repetidos con un rendimiento deficiente y/o deserción prematura.

Por lo anterior, en Chile existe una desigualdad educativa de base. Las Universidades, en gran medida, no se encuentran preparadas para implementar prácticas que aseguren óptimos resultados académicos ni la adecuada adquisición de competencias en los estudiantes. Las instituciones no están ofreciendo oportunidades según especificidades y requerimientos de los educandos, sino más bien, discriminan en la práctica a favor de unos y en desmedro de otros ${ }^{21}$.

Según manifiesta el Consejo Superior de Educación, las consecuencias de los problemas de rendimiento puede ser la brecha de conocimientos por debilidades en contenidos, escasos hábitos de estudio, metodologías de enseñanza y aprendizajes diferentes en Educación secundaria y terciaria, los de Colegios municipalizados son los que tendrían más debilidades académicas previas ${ }^{22}$.

Por todo lo antes mencionado, trabajar el aprendizaje de estrategias en las distintas etapas del sistema educativo es fundamental, ya que el uso de éstas influye de manera importante, junto con otros factores, en el rendimiento académico de los estudiantes ${ }^{23,24}$. Además, es imposible que los estudiantes puedan aprender todos los conocimientos, habilidades y actitudes que van a necesitar para su vida futura. Por ello, es necesaria la formación en habilidades para aprender a aprender, de manera autónoma y con eficacia, permanentemente, con herramientas que van a facilitar su progresivo desarrollo personal y profesional ${ }^{25}$.

Diversos estudios han encontrado que las estrategias de aprendizaje se relacionan de forma directa con la calidad de éste y permiten identificar las causas del nivel de rendimiento de los/as estudiantes ${ }^{26}$. Puede suceder que dos educandos con el mismo potencial intelectual, la misma motivación y receptores del mismo tipo de enseñanza tengan un rendimiento diferente debido a que utilizan estrategias de aprendizaje distintas.

$\mathrm{Al}$ respecto, los estudiantes con alto promedio académico tienden a emplear estilos activos y estrategias metacognitivas ${ }^{27}$, las mujeres a diferencia de los hombres obtienen mayor puntuación en las escalas de actitud, motivación, administración de tiempo, ayudas de estudio y autocomprobación ${ }^{28}$, ellas utilizan más estrategias de adquisición, recuperación y apoyo al procesamiento de la información, así como las de codificación, además de mayor uso de técnicas de organización ${ }^{9,29}$, también el género femenino obtiene un mayor rendimiento y una distribución más equilibrada de sus estilos y estrategias de aprendizaje dentro de una misma Carrera y entre las diferentes Carreras ${ }^{30}$. 
Estudios evidencian que los educandos con mejor rendimiento presentan mayor autoestima académica y familiar, suelen usar estrategias de aprendizaje más complejas ${ }^{31}$, al igual que aquellos expuestos al aprendizaje basado en problemas ${ }^{30}$. Existe una relación significativa entre rendimiento académico y uso de estrategias de aprendizaje, la motivación es el componente del aprendizaje estratégico que mejor predice el rendimiento académico ${ }^{32}$. Los que utilizan estrategias de aprendizaje más complejas presentan, significativamente, mejor rendimiento académico ${ }^{31}$ y también en aquellos que hacen uso de mejores y más estrategias ${ }^{25}$.

Las estrategias más utilizadas son la lectura y la relectura del material de estudio, a través de la selección de ideas importantes y la reorganización de la información ${ }^{33}$, las de elaboración y organización, antes que de repaso ${ }^{34}$. El estilo reflexivo y pragmático se correlaciona con alto rendimiento académico y con estrategias de aprendizaje profundas ${ }^{33}$.

Las debilidades en metodologías de enseñanza y aprendizaje ocasionan problemas de rendimiento académico, como también la brecha entre las exigencias de la Carrera y la formación base adquirida en años anteriores al ingreso a la Universidad, entre estas: debilidades en contenidos, escasos hábitos de estudios, metodologías de enseñanza y aprendizaje de la Universidad comparada con las de colegios, entre otras; la mejora en el rendimiento no es sólo producto del aumento en los contenidos ni de los cambios curriculares, sino también de la habilitación en destrezas intelectuales ${ }^{35}$.

Finalmente, se puede mencionar que la tasa de intento y la eficiencia se relacionan con el uso de estrategias que fomentan un aprendizaje significativo y autorregulado, el éxito, además se relaciona con un procesamiento cognitivo más profundo y con la búsqueda de aplicabilidad de los contenidos estudiados ${ }^{26}$

En el contexto de este estudio, la realidad de la Carrera de Enfermería, Universidad Santo Tomás (UST) a nivel central y local, no está ajena a los problemas descritos si se considera el perfil de estudiante que recibe, ya sea por el Proceso de Admisión Regular o el Especial. Para el primero el 80\% provienen de Liceos Científico Humanistas y Técnicos Profesionales Municipalizados o Particulares Subvencionados, promedio de Prueba de Selección Universitaria (PSU) en 500 puntos y nivel socioeconómico medio bajo y para el segundo no se considera puntaje PSU, pueden calificar titulados o estudiantes con al menos dos semestres cursados en otras Instituciones de Educación Superior, deportistas destacados y aquellos que hayan finalizado su Enseñanza Media en el extranjero ${ }^{36}$.

En cuanto a la deserción de estudiantes, de acuerdo al Informe de Gestión Primer Semestre, existen las de Retiro temporal: baja carga horaria para el semestre o imposibilidad de inscribir asignaturas en el semestre lectivo debido a reprobación de prerrequisitos; un porcentaje menor se debe a embarazos o problemas de salud; Retiro definitivo: bajo rendimiento académico o embarazo o cambio de Sede (traslado de domicilio y causales económicas). La Tasa de retención en los dos primeros años se encuentra sobre el promedio histórico nacional. Respecto al Tiempo de egreso, a nivel local el tiempo promedio es de 12.9 semestres, es decir, 2.9 semestres por sobre la duración teórica esperada de la Carrera que es de 10 semestres $^{36}$.

Dentro de las limitaciones de los estudiantes destaca, a juicio de los docentes de Enfermería, que en general se observan con inadecuadas técnicas y hábitos de estudio, dificultades para tomar apuntes, limitaciones en la expresión oral y escrita, poca inclinación a la lectura, cultura general limitada a sus intereses muy personales.

Específicamente, en virtud de la relevancia que tienen las estrategias de aprendizaje para la mejora de los indicadores antes mencionados, y frente a la inexistencia de investigaciones en este foco, se realizó un estudio no experimental de corte transversal durante el segundo semestre de 2015, cuyo objetivo fue: relacionar las estrategias de aprendizaje de los estudiantes de la Carrera de Enfermería de la Universidad Santo Tomás sede Temuco, con el rendimiento académico y variables sociodemográficas. En tanto los específicos se orientaron a: a) caracterizar sociodemográficamente la población en estudio: sexo, edad, tipo de colegio y tipo de formación, b) determinar el rendimiento académico con base en: el número 
de asignaturas reprobadas, promedio general acumulado, y notas de enseñanza media, c) identificar las estrategias de aprendizaje que utilizan los alumnos d) comparar los factores y dimensiones de las estrategias de aprendizaje según sexo, edad, colegio y tipo de colegio, asignaturas reprobadas y promedio ponderado.

La importancia de realizar esta investigación radica en que la mejora en el rendimiento no es sólo producto del aumento en los contenidos ni de los cambios curriculares, sino también de la habilitación en destrezas intelectuales, lo cual se logra con estrategias que faciliten el aprendizaje de destrezas cognitivas y que fomenten el aprendizaje autónomo ${ }^{37}$. Por otra parte, al entender las formas de procesamiento de la información, es posible mejorar el rendimiento académico, producto del incremento de la efectividad académica y del consiguiente aprendizaje significativo, que implica comprender, relacionar la nueva información con inclusores previos, interacción con los contenidos y relación con lo cotidiano ${ }^{38,39}$.

\section{Métodos}

Se trata de una investigación no experimental de corte transversal, y de acuerdo con su finalidad, a un estudio correlacional, ya que asocia variables, para establecer la relación que se generan entre ellas ${ }^{40,41}$.

La muestra se constituyó por Estudiantes de Enfermería de la Universidad Santo Tomás-Temuco de todos los niveles de la Carrera. Fue no probabilística, de conveniencia o por selección intencionada ${ }^{42}$, conformada por 185 (de un total de 232) matriculados en el segundo semestre, año 2015 y que se encontraban presentes en el momento de la aplicación del instrumento.

Se utilizó el instrumento Escala de Estrategias de Aprendizaje ACRA-Abreviada (Adquisición, Codificación, Recuperación y Apoyo de la Información), para estudiantes universitarios ${ }^{45}$, el cual fue aplicado durante noviembre del año 2015, previa autorización de los Directivos y docentes de la Carrera y Consentimiento Informado de las/os estudiantes.

Es un instrumento de autoinforme, publicado en castellano y basado en los principios del procesamiento de la información de la teoría cognitiva ${ }^{43}$. Toma la denominación de Abreviada a partir de su elaboración de acuerdo a la original ${ }^{9}$, destinada a alumnos secundarios. Consta de 44 ítems, cada uno de los cuales enuncia una estrategia de aprendizaje posible de ser utilizada por estudiantes universitarios. Se responde de acuerdo con una escala Likert de cuatro opciones que va de la menos a la más utilizada. El conjunto total de ítems ha sido seleccionado en función de tres dimensiones y 13 factores que intervienen en la adquisición de la información, la codificación, la recuperación y el apoyo: para su validación se efectuó un ANOVA entre el uso de estrategias de aprendizaje y el nivel de rendimiento académico. Los índices de fiabilidad de la Escala ACRA-Abreviada para estudiantes universitarios son aceptables, con un alpha global de $0.8828^{44-46}$.

Con los datos obtenidos se constituyó una base de datos en Excel; el procesamiento de la información se realizó con el Programa Stata 11.0.

Se realizaron estadísticas descriptivas en tablas, gráfico de puntos, se obtuvieron medidas de tendencia central y varianza. Para la comparación de los promedios se usó la prueba t-test y ANOVA, según corresponda, la relación entre variables cuantitativas con el coeficiente de correlación de Pearson. Nivel de significación utilizado fue de 5\%.

Inicialmente, se realizó la descripción estadística sociocultural de la muestra, luego se detallaron académicamente a los estudiantes. Aquí se consideraron aspectos académicos previos al ingreso a la Universidad y de desempeño durante su Carrera. Posterior a ello, se describió el grado de uso de estrategias de aprendizaje a través del cuestionario Escala de Estrategias de Aprendizaje ACRA-Abreviada ${ }^{43}$, aplicada a 185 estudiantes, la cual se respondió de acuerdo con una escala Likert de cuatro opciones, 0 (nunca), 1 (a veces), 2 (bastantes veces) y 3 (siempre), se tomó como punto de corte una puntuación superior al $75 \%$ de su utilización académica. 
A continuación, se aplicaron las pruebas estadísticas de correlación entre el uso de Estrategias de aprendizaje y Rendimiento académico, expresados en el Promedio, Asignaturas reprobadas, tipo de Formación de enseñanza media, tipo de Colegio de procedencia y Edad.

Los aspectos éticos fueron resguardados de la siguiente forma: solicitud de autorización a investigadores de la Escala ACRA-Abreviada mediante correo electrónico enviado al autor, aprobación y solicitud de permisos a autoridades correspondientes de la Institución, se comunicaron los fines de estudio, y Formulario de Consentimiento Informado, que garantiza el anonimato y la confidencialidad de los datos ${ }^{47}$.

\section{Resultados}

Los resultados se presentan en relación con los objetivos del estudio. La caracterización sociodemográfica de la población en estudio según sexo, edad, tipo de colegio y tipo de formación, se muestran en la Tabla 1, donde se observa que la mayoría son mujeres, provienen de colegios municipales y de formación científico humanista.

Tabla 1. Características sociodemográficas

\begin{tabular}{lcc}
\hline \multicolumn{1}{l}{ Sexo } & n & \% \\
\hline Mujer & 151 & 81.6 \\
Hombre & 34 & 18.4 \\
\hline Tipo de Colegio de procedencia & 10 & \\
\hline Particular & 81 & 43.8 \\
Particular subvencionado & 94 & 50.8 \\
Municipal & & \\
\hline Tipo de formación previa & 153 & 82.7 \\
\hline Científico humanista & 32 & 17.3 \\
Técnico profesional & $23.8 \pm 3.87$ & \\
Edad (media \pm DE) & 18 y 44 & \\
Mín. y máx. &
\end{tabular}

Con respecto al segundo objetivo, se puede mencionar que el número de asignaturas reprobadas, promedio general acumulado y notas de enseñanza media, los estudiantes presentan entre 0 y 20 asignaturas reprobadas. El $16.2 \%$ de ellos va al día respecto a su avance curricular, es decir, no ha repetido ninguna asignatura en todos sus años de Carrera. El 71.3\%, presentan dos o más asignaturas reprobadas, dato importante a considerar cuando se establecen los indicadores de casos críticos. En relación con el Promedio General Acumulado que presentan los estudiantes de la Carrera, los datos muestran que el mínimo corresponde a un 4.2, mientras que el máximo alcanzado es un 6.0 , con un promedio de $5.1 \pm 0.33$ (escala de notas de 1 a 7 ).

En la Tabla 2 se presentan los Factores de la Dimensión I Estrategias cognitivas y de Control del aprendizaje, en relación a las estrategias de aprendizaje de la escala ACRA abreviada. Se aprecia que en el Factor I: Selección y organización, el promedio de las frecuencias más alto es un 2.0, que corresponde al ítem 6 Antes de responder a un examen evoco aquellos agrupamientos de conceptos (resúmenes, esquemas, secuencias, diagramas, mapas conceptuales) realizados a la hora de estudiar. En tanto en el Factor II: Conciencia de la funcionalidad de las estrategias se presenta el ítem 14 con un 2.3, He caído en la cuenta que es beneficioso (cuando necesito recordar informaciones para un examen, trabajo, etc.) buscar en mi memoria las nemotecnias, dibujos, mapas conceptuales, etc., que elaboré al estudiar. En el Factor III: Estrategias de elaboración es un 2.2 para el ítem 17, Me ayuda a recordar lo aprendido el evocar sucesos, episodios o anécdotas (es decir "claves"), ocurridos durante la clase o en otros momentos del aprendizaje. En el Factor V: Planificación y 
control de la respuesta en situación de evaluación es un 2.3 para el ítem 19, Frente a un problema o dificultad considero, en primer lugar, los datos que conozco antes de aventurarme a dar una solución intuitiva. En el Factor VII: Subrayado es un 2.3 para el ítem 7, En los libros, apuntes u otro material a aprender, subrayo en cada párrafo las palabras, datos o frases que me parecen más importantes. En el Factor IX: Repetición y relectura es un 2.6 para el ítem 25, Cuando el contenido de un tema es denso y difícil vuelvo a leerlo despacio, éste es el que tiene el más alto promedio de las frecuencias de todos los factores de esta Dimensión.

Tabla 2. Factores de la Dimensión I Estrategias cognitivas y de Control del aprendizaje, n=185 ( Porcentajes)

\begin{tabular}{|c|c|c|c|c|c|c|}
\hline & Nunca & A veces & $\begin{array}{c}\text { Bastantes } \\
\text { veces }\end{array}$ & Siempre & & \\
\hline Ítem & $\%$ & $\%$ & $\%$ & $\%$ & Prom & DS \\
\hline \multicolumn{7}{|c|}{ Factor I: Selección y organización } \\
\hline 1 & 7.0 & 28.6 & 36.7 & 27.5 & 1.8 & 0.9 \\
\hline 2 & 12.4 & 42.1 & 31.3 & 14.0 & 1.4 & 0.9 \\
\hline 3 & 7.0 & 35.3 & 32.6 & 24.8 & 1.6 & 0.9 \\
\hline 4 & 13.5 & 28.1 & 33.5 & 24.4 & 1.6 & 1.0 \\
\hline 5 & 2.7 & 33.7 & 44.5 & 19.0 & 1.7 & 0.8 \\
\hline 6 & 4.3 & 18.9 & 41.0 & 35.6 & 2.0 & 0.8 \\
\hline Total & & & & & 1.8 & 0.6 \\
\hline \multicolumn{7}{|c|}{ Factor II: Conciencia de la funcionalidad de las estrategias } \\
\hline 11 & 2.1 & 13.6 & 43.1 & 40.9 & 2.2 & 0.8 \\
\hline 12 & 3.8 & 20.2 & 55.1 & 20.7 & 1.9 & 0.7 \\
\hline 13 & 0.5 & 15.7 & 43.8 & 40.0 & 2.2 & 0.7 \\
\hline 14 & 2.7 & 11.3 & 36.7 & 49.1 & 2.3 & 0.8 \\
\hline 15 & 4.8 & 28.2 & 43.4 & 23.3 & 1.8 & 0.8 \\
\hline Total & & & & & 2.1 & 0.6 \\
\hline \multicolumn{7}{|c|}{ Factor III: Estrategias de elaboración } \\
\hline 16 & 3.2 & 20.5 & 37.4 & 38.9 & 2.1 & 0.9 \\
\hline 17 & 1.6 & 15.8 & 37.8 & 44.8 & 2.2 & 0.8 \\
\hline 18 & 2.7 & 17.3 & 41.0 & 38.9 & 2.1 & 0.8 \\
\hline Total & & & & & 2.2 & 0.6 \\
\hline \multicolumn{7}{|c|}{ Factor V: Planificación y control de la respuesta en situación de evaluación } \\
\hline 19 & 0.5 & 10.8 & 42.7 & 45.9 & 2.3 & 0.7 \\
\hline 20 & 8.7 & 29.7 & 31.8 & 29.7 & 1.8 & 1.0 \\
\hline 21 & 4.4 & 30.3 & 47.5 & 17.8 & 1.7 & 0.8 \\
\hline 22 & 2.2 & 14.2 & 43.4 & 40.1 & 2.2 & 0.8 \\
\hline 23 & 1.0 & 15.2 & 39.2 & 44.5 & 2.2 & 0.8 \\
\hline Total & & & & & 2.1 & 0.5 \\
\hline \multicolumn{7}{|c|}{ Factor VII: Subrayado } \\
\hline 7 & 3.2 & 15.2 & 30.2 & 51.4 & 2.3 & 0.8 \\
\hline 8 & 3.8 & 14.8 & 32.2 & 49.2 & 2.2 & 0.9 \\
\hline 9 & 18.9 & 14.6 & 19.5 & 47.0 & 1.9 & 1.2 \\
\hline 10 & 10.8 & 15.1 & 27.0 & 47.0 & 2.1 & 1.0 \\
\hline Total & & & & & 2.1 & 0.7 \\
\hline \multicolumn{7}{|c|}{ Factor IX: Repetición y relectura } \\
\hline 24 & 1.0 & 12.0 & 31.8 & 55.2 & 2.4 & 0.7 \\
\hline 25 & 1.6 & 4.4 & 21.7 & 72.2 & 2.6 & 0.6 \\
\hline Total & & & & & 2.5 & 0.6 \\
\hline
\end{tabular}


En la Tabla 3 se dan a conocer los Factores de la Dimensión II Estrategias de apoyo al aprendizaje en relación a las estrategias de aprendizaje de la escala ACRA abreviada. Se aprecia que en el Factor IV: Motivación intrínseca, los promedios de las frecuencias más altas son de un 2.3, que corresponden a los ítems 27 y 29, respectivamente, Me esfuerzo en el estudio para sentirme orgulloso de mí mismo y me digo a mi mismo que puedo superar mi nivel de rendimiento actual (expectativas) en las distintas asignaturas. En tanto en Factor VIII: Apoyo social se presenta el ítem 36 con un 2.6, Me satisface que mis compañeros, profesores y familiares valoren positivamente mi trabajo. En el Factor X: Horario y plan de trabajo es un 2.1 para el ítem 38, Antes de iniciar el estudio, distribuyo el tiempo de que dispongo entre todos los temas que tengo que aprender. En el Factor XI: Condiciones contra distractoras es un 2.0 para el ítem 31, Procuro que en el lugar de estudio no haya nada que pueda distraerme, como personas, ruidos, desorden, falta de luz, ventilación, etc. En el Factor XIII: Control de la ansiedad es un 2.0 para el ítem 30, Pongo en juego recursos personales para controlar mis estados de ansiedad cuando me impiden concentrarme en el estudio. El Factor que tiene el más alto promedio de las frecuencias de todos los factores de esta Dimensión es el Apoyo social con un 2.3.

Tabla 3. Factores de la Dimensión II Estrategias de apoyo al aprendizaje $n=185$ (Porcentajes)

\begin{tabular}{|c|c|c|c|c|c|c|}
\hline & Nunca & A veces & Bastantes veces & Siempre & & \\
\hline Ítem & $\%$ & $\%$ & $\%$ & $\%$ & Prom & $\overline{\text { DS }}$ \\
\hline \multicolumn{7}{|c|}{ Factor IV: Motivación intrínseca } \\
\hline 26 & 2.2 & 15.8 & 42.3 & 39.6 & 2.1 & 0.77 \\
\hline 27 & 3.2 & 9.7 & 35.3 & 51.6 & 2.3 & 0.79 \\
\hline 28 & 5.9 & 14.1 & 35.3 & 44.5 & 2.1 & 0.89 \\
\hline 29 & 3.8 & 12.5 & 32.3 & 51.3 & 2.3 & 0.84 \\
\hline Total & & & & & 2.2 & 0.64 \\
\hline \multicolumn{7}{|c|}{ Factor VIII: Apoyo social } \\
\hline 33 & 5.4 & 11.9 & 34.7 & 47.8 & 2.2 & 0.9 \\
\hline 34 & 2.8 & 13.5 & 35.8 & 47.8 & 2.2 & 0.8 \\
\hline 35 & 1.7 & 17.4 & 43.4 & 37.4 & 2.1 & 0.8 \\
\hline 36 & 1.0 & 4.9 & 23.4 & 70.7 & 2.6 & 0.6 \\
\hline 37 & 1.0 & 6.0 & 25.7 & 67.3 & 2.5 & 0.7 \\
\hline Total & & & & & 2.3 & 0.5 \\
\hline \multicolumn{7}{|c|}{ Factor X: Horario y plan de trabajo } \\
\hline 38 & 4.9 & 19.2 & 37.7 & 38.2 & 2.1 & 0.9 \\
\hline 39 & 5.4 & 26.7 & 39.8 & 27.8 & 1.9 & 0.9 \\
\hline Total & & & & & 2.0 & 0.8 \\
\hline \multicolumn{7}{|c|}{ Factor XI: Condiciones contra distractoras } \\
\hline 31 & 11.9 & 18.5 & 24.4 & 45.0 & 2.0 & 1.0 \\
\hline 32 & 16.8 & 30.5 & 26.7 & 26.0 & 1.6 & 1.0 \\
\hline Total & & & & & 1.8 & 0.8 \\
\hline \multicolumn{7}{|c|}{ Factor XIII: Control de la ansiedad } \\
\hline 30 & 3.2 & 21.3 & 43.1 & 32.2 & 2.0 & 0.8 \\
\hline Total & & & & & 2.0 & 0.8 \\
\hline
\end{tabular}

En la Tabla 4 se presentan los factores de la Dimensión III, Hábitos de estudio con relación a las estrategias de aprendizaje de la escala ACRA abreviada. Se observa que en el Factor VI: Comprensión, todos los ítems 40, 41 y 42 tienen un promedio de 2.3, en lo que concierne a, Intento expresar lo aprendido con mis propias palabras, en vez de repetir literalmente o al pie de la letra lo que dice el libro o profesor, Procuro aprender los temas con mis propias palabras en vez de memorizarlos al pie de la letra y cuando estudio trato de resumir mentalmente lo más importante respectivamente. En tanto el Factor XII: Hábitos de estudio, el promedio más alto corresponde al ítem 44, Cuando estoy estudiando una lección, para facilitar la comprensión, descanso y después la repaso para aprenderla mejor. 
Tabla 4. Factores de la Dimensión III Hábitos de estudio

\begin{tabular}{|c|c|c|c|c|c|c|}
\hline & Nunca & A veces & Bastantes veces & Siempre & & \\
\hline Ítem & $\%$ & $\%$ & $\%$ & $\%$ & Prom & DS \\
\hline \multicolumn{7}{|c|}{ Factor VI: Comprensión } \\
\hline 40 & 0.5 & 8.1 & 43.7 & 47.5 & 2.3 & 0.65 \\
\hline 41 & 0.5 & 9.8 & 41.8 & 47.5 & 2.3 & 0.67 \\
\hline 42 & 0.5 & 11.8 & 43.2 & 44.4 & 2.3 & 0.71 \\
\hline Total & & & & & 2.3 & 0.54 \\
\hline \multicolumn{7}{|c|}{ Factor XII: Hábitos de estudio } \\
\hline 43 & 8.1 & 26.4 & 36.3 & 29.9 & 1.8 & 0.92 \\
\hline 44 & 3.3 & 12.9 & 42.2 & 41.6 & 2.2 & 0.79 \\
\hline Total & & & & & 2.0 & 0.7 \\
\hline
\end{tabular}

La comparación de los factores y dimensiones de las estrategias de aprendizaje según sexo y edad se presentan en la Tabla 5. Se observa que las puntuaciones promedio del Factor I: Selección y organización, son diferentes entre mujeres y hombres, éstas son mayores en las primeras, concentrándose en la respuesta bastantes veces. La misma tendencia se observa para el Factor III: Estrategias de elaboración y VII: Subrayado. En los Factores II: Conciencia de la funcionalidad de las estrategias, V: Planificación y control de la respuesta en situación de evaluación y IX: Repetición y relectura, no se encontraron diferencias significativas, pero sus respuestas se centralizan en bastantes veces y siempre. En la Dimensión I: Estrategias cognitivas y de control del aprendizaje, se observa que en términos globales, existen diferencias significativas en las puntuaciones promedios entre mujeres y hombres, las cuales son mayores en mujeres, la tendencia de respuestas están en la categoría de bastantes veces. En la Dimensión II: Estrategias de apoyo al aprendizaje, ningún Factor presenta diferencias en sus puntuaciones promedios entre mujeres y hombres, ni en la Dimensión total, la tendencia de las respuesta es hacia bastantes veces. Similar comportamiento se observa en la Dimensión III: Hábitos de estudio, donde ninguno de sus Factores muestra una diferencia significativa en las puntuaciones entre mujeres y hombres, ni en la Dimensión total. De igual manera, la tendencia de respuesta es hacia bastantes veces. Según edad, no se aprecian relaciones significativas en ninguna de las tres dimensiones.

Tabla 5: Comparación de los Factores y Dimensiones de las estrategias de aprendizaje según sexo y edad

\begin{tabular}{|c|c|c|c|c|c|c|c|}
\hline & \multicolumn{4}{|c|}{ Sexo } & & \multicolumn{2}{|c|}{ Edad } \\
\hline & \multicolumn{2}{|c|}{ Mujer (n=151) } & \multicolumn{2}{|c|}{ Hombre $(n=34)$} & \multirow[b]{2}{*}{$\mathbf{p}$} & \multirow[b]{2}{*}{$\mathbf{r}$} & \multirow[b]{2}{*}{$\mathbf{p}$} \\
\hline & & Media \pm DS & & Media \pm DS & & & \\
\hline Dimensión I & 2.1 & 0.35 & 1.8 & 0.33 & $0.0011^{*}$ & 0.0165 & 0.8254 \\
\hline F I & 1.8 & 0.57 & 1.5 & 0.64 & $0.0105^{*}$ & -0.0277 & 0.7118 \\
\hline F II & 2.1 & 0.52 & 2.0 & 0.48 & $0.4127^{*}$ & 0.0356 & 0.6338 \\
\hline F III & 2.2 & 0.58 & 1.9 & 0.65 & $0.0190 *$ & -0.0658 & 0.3792 \\
\hline $\mathrm{FV}$ & 2.1 & 0.51 & 2.0 & 0.40 & $0.2434^{*}$ & -0.006 & 0.9356 \\
\hline F VII & 2.2 & 0.66 & 1.7 & 0.74 & $0.0008^{*}$ & 0.0822 & 0.2714 \\
\hline F IX & 2.5 & 0.57 & 2.4 & 0.54 & $0.4017 *$ & 0.0473 & 0.5285 \\
\hline Dimensión II & 2.2 & 0.46 & 2.1 & 0.45 & $0.5925 *$ & 0.0408 & 0.5855 \\
\hline F IV & 2.2 & 0.63 & 2.2 & 0.67 & $0.9179 *$ & 0.1035 & 0.1667 \\
\hline F VIII & 2.4 & 0.49 & 2.2 & 0.59 & $0.2208^{*}$ & -0.0954 & 0.2028 \\
\hline $\mathrm{FX}$ & 2.0 & 0.80 & 1.9 & 0.72 & $0.8698^{*}$ & 0.0102 & 0.8918 \\
\hline F XI & 1.8 & 0.81 & 1.8 & 0.88 & $0.7309 *$ & 0.1167 & 0.1187 \\
\hline F XIII & 2.0 & 0.81 & 1.9 & 0.85 & $0.4192 *$ & -0.0132 & 0.8612 \\
\hline Dimensión III & 2.2 & 0.48 & 2.2 & 0.37 & $0.8583^{*}$ & 0.0328 & 0.673 \\
\hline FVI & 2.3 & 0.54 & 2.4 & 0.51 & $0.3674^{*}$ & 0.0007 & 0.9925 \\
\hline F XII & 2.0 & 0.73 & 1.8 & 0.54 & $0.1128 * *$ & 0.0614 & 0.4113 \\
\hline
\end{tabular}

*ANOVA **test para varianzas distintas 
Respecto al tipo de Colegio de procedencia de los estudiantes (Científico humanista, Técnico profesional), se aprecia que en la Dimensión I, sólo el Factor II: Conciencia de la funcionalidad de las estrategias, presenta diferencias significativas en las puntuaciones promedios, estas son mayores en los que provienen de colegios Científico humanista, la tendencia de respuesta de ese Factor apunta hacia la categoría bastantes veces. No se aprecian diferencias significativas entre los Factores I: Selección y organización, III: Elaboración, V: Planificación y control de la respuesta en situación de evaluación, VII: Subrayado y IX: Repetición y relectura, las cuales concentran sus respuestas entre a veces y siempre. La Dimensión I, en su globalidad, presenta tendencia promedio hacia la respuesta bastantes veces, no encontrándose diferencias significativas por formación. Los Factores pertenecientes a la Dimensión II: Motivación intrínseca, Control de la ansiedad, Condiciones contra distractoras, Apoyo social, Horario y Plan de trabajo, no presentan diferencias en sus puntuaciones promedios, las respuestas se inclinan hacia bastantes veces. El mismo comportamiento se aprecia para la Dimensión III.

Con respecto a la comparación de los factores y dimensiones de las estrategias de aprendizaje según asignaturas reprobadas y promedio ponderado a las asignaturas reprobadas, se observa en las tres Dimensiones, que el promedio de respuestas se concentra en bastantes veces, similar entre los que no reprobaron y los que reprobaron al menos una asignatura. Además, de considerar el promedio ponderado, existe una relación lineal baja entre éste y la Dimensión I. El mismo comportamiento se observa en la Dimensión II.

\section{Discusión}

Todos los datos sociodemográficos, son similares a los de nivel nacional, según Informe Global de Autoevaluación, Universidad Santo Tomás, Santiago de Chile, 2009 y tienen relación con el perfil socioeconómico de la gran cantidad de los educandos, los cuales según este mismo Informe se encuentran en los tres primeros quintiles a nivel nacional: 7\% en el primer quintil (A); $25 \%$ en el segundo (B) y el $46 \%$ en el tercero (C), lo que significa un bajo perfil socioeconómico y que coincide con el sello Institucional.

Según el Informe de Gestión Primer Semestre, Universidad Santo Tomás, sede Temuco 2015, el que no hayan egresado 39 estudiantes y que el $71.3 \%$ hayan reprobado una o más asignaturas, se podría generar, por varios factores, entre ellos: existe un porcentaje no menor que presentan dificultades económicas, con la necesidad de trabajar para financiar su formación, lo que incide en una menor dedicación a los estudios, aumento de la repitencia y eliminación reglamentaria. Por otra parte, también existe un número de discentes que permanece en la carrera, ya que han debido realizar retiros temporales por distintas causas, algunas de las cuales son académicas, ya que por reprobación de pre requisitos no tienen actividades curriculares que cursar en determinados semestres.

Respecto al Promedio General Acumulado, se puede apreciar que es aproximadamente de 5.1, con notas que van desde 4.2 el más bajo y 6.0 el más alto. En muchas ocasiones se ve favorecido al considerar aquellas asignaturas que son electivas y en las cuales generalmente obtienen buenas calificaciones. Sin embargo, se debe recordar que para determinar el rendimiento es importante considerar el Promedio General Acumulado; además de atender los indicadores: Tasas de aprobación, Tasas de retención, Deserción académica, Repitencia, Tasa de titulación oportuna, pues es probable que un estudiante presente un promedio aceptable o bueno, no obstante, ha repetido más de una asignatura, ha postergado sus estudios, por tanto, aumenta su tiempo de permanencia. En este aspecto se postula que los indicadores anteriormente mencionados, reflejan los resultados del trabajo académico y expresan la eficiencia y calidad con la que directivos, profesores y estudiantes interactúan en el proceso de enseñanza-aprendizaje ${ }^{12-14}$. De acuerdo a esto, no se puede afirmar que los participantes de este estudio tienen un buen rendimiento académico, pues a pesar de que el Promedio General Acumulado tiene una media suficiente (5.1), hay un porcentaje importante que han reprobado más de una asignatura, por lo que la permanencia en la Universidad se ha extendido en algunos casos por más de 9 años.

Los resultados dan cuenta que las tres Dimensiones tienen un promedio de utilización muy similar, la Dimensión I, presenta sólo una décima de diferencia respecto de la II, y la III. 
En su totalidad los Factores más valorados son: IX (Repetición y relectura), VIII (Apoyo social) y el VI (Comprensión), lo que coincide con una investigación-intervención con alumnos universitarios, donde las estrategias más utilizadas fueron la lectura y la relectura del material de estudio ${ }^{33}$.

Según la clasificación de estrategias de aprendizaje de la escala ACRA ${ }^{9}$, y además de considerar los factores pertenecientes a cada dimensión, se puede resumir que las estrategias que ocupan en mayor medida los estudiantes corresponden a las de Adquisición de la información, a través de la repetición, las cuales les permiten llevar la información del registro sensorial a la memoria a corto plazo ${ }^{9}$, por lo que favorecen un aprendizaje más bien memorístico. En segundo lugar utilizan las de Apoyo al procesamiento de la información, de tipo Socioafectivas. Si bien, éstas son importantes, puesto que, si se refuerza la motivación, la autoestima y la atención, se garantiza el clima adecuado para un buen funcionamiento de todo el sistema cognitivo, no se dirigen directamente al aprendizaje de los contenidos. La misión fundamental de estas estrategias apuntan sólo a mejorar la eficacia del aprendizaje al optimizar las condiciones en las que se produce ${ }^{9}$. En tercer lugar, están las de Codificación de información, que permiten el paso de la información de la memoria de corto plazo a la de largo plazo, precisan de más tiempo y esfuerzo para que la información sea almacenada a largo plazo, y faciliten de esta manera la significación de los contenidos.

En relación a la distinción de acuerdo a sexo, las mujeres que participaron en este estudio ocupan mayor número de estrategias pertenecientes a la Dimensión I, las cuales corresponden a las de Selección y organización (codificación), Estrategias de elaboración (codificación) y de Subrayado (adquisición). No se observaron diferencias entre hombres y mujeres en las otras dimensiones, lo que es coincidente de alguna manera con otros estudios donde se plantea que las féminas utilizan mayor número de estrategias de aprendizaje de adquisición, codificación, recuperación, y apoyo ${ }^{28-30}$.

No hubo correlación con la edad. Se creyó importante establecer esta relación ya que se podría esperar que a medida que el estudiante va avanzando curricularmente, vaya adquiriendo mayores herramientas para enfrentarse al proceso de enseñanza aprendizaje. De igual forma, es interesante establecer el perfil de estrategias con las que el estudiante ingresa a la Universidad, pues la masificación del acceso a la educación terciara, implica una población estudiantil más grande y diversa, lo cual trae consigo muchas veces que ingresen alumnos sin las competencias mínimas que requiere el Plan de Estudio ${ }^{19}$, postulándose además que la gran mayoría de los educandos no están adecuadamente preparados para lo que se requiere de ellos en la Universidad, más aún, muchas veces no son capaces de autorregular su propio proceso de aprendizaje ${ }^{13}$. En el estado del arte no se encontraron investigaciones que correlacionen la edad con las estrategias, por lo que no se pudo contrastar.

Al comparar los Factores y Dimensiones de las estrategias de aprendizaje según la formación del Colegio, se aprecia que sólo el Factor II (Conciencia de la funcionalidad de las estrategias) presenta diferencias significativas en las puntuaciones promedios, las cuales son mayores en los que provienen de Colegios Científico Humanistas. Todos los ítems que conforman este Factor, se relacionan con las estrategias de apoyo al procesamiento de la información. La Dimensión II y III no presenta diferencias, por lo tanto en términos generales, no se puede afirmar que la formación que traen los estudiantes de enseñanza media, influye en la adquisición de estrategias de aprendizaje.

En relación con el tipo de colegio del cual provienen los/as estudiantes, la única Dimensión que presenta diferencias, corresponde a la III, los estudiantes que provienen de Particulares subvencionados y Municipalizados presentan mayor utilización de estrategias de comprensión y de hábitos de estudio. Llama la atención este hallazgo pues contrasta con los datos aportados por MINEDUC ${ }^{22}$, que plantea que las consecuencias de los problemas de rendimiento universitario, puede ser la brecha de conocimientos por debilidades en contenidos, escasos hábitos de estudio, metodologías de enseñanza y aprendizajes diferentes en Educación secundaria y terciaria, son los de Colegios municipalizados, los que tendrían más debilidades académicas previas. 
Así mismo, si se consideran indicadores de rendimiento académico, como el número de asignaturas reprobadas, se observa que no hay relación entre el uso de estrategias y la reprobación de ellas, ya que la distribución de éstas es similar para cada una de las Dimensiones.

Para el Promedio General Acumulado, existen relaciones en la Dimensión I, específicamente en los Factores II (Conciencia de la funcionalidad de las estrategias), III (Estrategias de elaboración), VII (Subrayado), en la Dimensión II, con el Factor IV (Motivación intrínseca), VIII (Apoyo social), X (Horario y plan de trabajo), XI (Condiciones contra distractoras), XIII (Control de la ansiedad), sin embargo, esta relación es baja, es decir, la correlación de Pearson fue lineal positiva, pero muy cercanas a cero (van de 0.1553 a 0.2281 ), por lo que el porcentaje que tienen en común estas estrategias con la calificación registrada por los/las estudiantes van de un $2.4 \%$ a un $5.2 \%$, respectivamente.

De acuerdo a los resultados mostrados anteriormente, los/las estudiantes conocen y movilizan un número importante de estrategias de aprendizaje, aunque, no se pudo demostrar consistentemente que el uso de ellas influya en los indicadores de rendimiento académico, como se ha evidenciado en múltiples estudios relacionados a esta temática ${ }^{13-15,25-27,30-32}$.

Un elemento necesario de considerar y que podría explicar los resultados, hace referencia al tipo de evaluación a la que se someten los/as estudiantes. Por lo general éstas tienden a estancarse en modelos que exigen por parte de los educandos la memorización y el aprendizaje mecánico, por lo tanto impide que el aprendizaje se elabore en procesos cognitivos superiores. Esto hace que sea difícil valorar las estrategias de aprendizaje que emplean, y que éstas verdaderamente tengan consecuencias en su rendimiento académico, también explica el hecho que ocupen más estrategias de repetición.

Es importante considerar que cada tipo de aprendizaje estaría vinculado a una serie de estrategias que le son propias. El uso de ellas, por tanto, dependerá de algunos factores como los contenidos de aprendizaje (tipo y cantidad), los conocimientos previos que tenga sobre el contenido de aprendizaje, las condiciones de aprendizaje (tiempo disponible, las ganas de estudiar, etc.), y el tipo de evaluación al que va a ser sometido ${ }^{30}$. De ahí, la importancia que tienen los métodos de evaluación para favorecer en forma efectiva el uso de estrategias de aprendizaje de los estudiantes. Si no se promueve la evaluación auténtica de los aprendizajes, y sólo se limitan estos procesos a la reproducción de contenidos, el educando se condicionará a usar recursos que únicamente movilicen la repetición de información, un aprendizaje memorístico, pues los métodos de evaluación a los que permanentemente se someten no promueven la aplicación de los contenidos ni el aprendizaje significativo.

De acuerdo a lo anterior, es de vital importancia el rol del docente como mediador para promover la capacidad en los educandos de gestionar su aprendizaje, de hacerlos responsables y entes activos de su propia educación, así como comenzar desde los primeros niveles de pregrado para dotarlos de las herramientas intelectuales y sociales que hagan posible su desarrollo continuo en el posgrado. Para ello, es necesario preparar a los académicos en la enseñanza de estrategias de aprendizaje y en los métodos de evaluación que promuevan la utilización de éstas.

No obstante, que los resultados no hayan sido concordantes con la mayoría de los estudios en los cuales se correlacionan de manera positiva las estrategias y el rendimiento, es clara la importancia que éstas tienen como herramientas para la adquisición de competencias, que les permitan a los/as estudiantes ser autónomos a la hora de enfrentarse a las demandas de los nuevos aprendizajes que asumirán a lo largo de su vida. Además, promueven que dichos aprendizajes sean significativos, dándoles la autonomía para aprender a aprender.

\section{Conclusión}

Se puede concluir en el presente estudio que no existe una correlación significativa entre el uso de estrategias de aprendizaje y el rendimiento académico, lo cual indica que utilizar un número importante 
de estrategias no garantiza el éxito académico. Los/as estudiantes de enfermería de la Universidad Santo Tomás, sede Temuco, conocen un número importante de estrategias y hacen uso de ellas, pero no de una manera profunda, empleándolas básicamente como elementos de apoyo al aprendizaje y memorización, ya que las más usadas corresponden a las de repetición y relectura y de apoyo, las cuales no promueven el aprendizaje significativo.

\section{Responsabilidades éticas}

Protección de personas y animales. Los autores declaran que para esta investigación no se han realizado experimentos en seres humanos ni en animales.

Confidencialidad de los datos. Declaran que en este artículo no aparecen datos de estudiantes.

Derecho a la privacidad y consentimiento informado. Los autores han obtenido el consentimiento informado de los sujetos referidos en el artículo.

Conflicto de intereses. Ninguno.

\section{Referencias}

1. Jacobs B, Van der Ploeg F. Guide to reform of higher education: a European perspective. Econ. Policy. 2006; 21(47): 535-92. [Consultado junio 28 2015]. Disponible en: https://bit.ly/2HedRBV

2. Herrera-Torres L, Enrique-Mirón C. Proyectos de Innovación en Tutorías en la Universidad de Granada: Análisis de los instrumentos empleados. Profr. rev. curríc. form. profr. 2008; 12(2): 1-18. [Consultado julio 10 2015]. Disponible en: https://bit.ly/2SKedBe

3. Bajo MT, Maldonado A, Moreno S, Moya A. Las competencias en el nuevo paradigma educativo para Europa. Granada: Vicerrectorado de Planificación Calidad y Evaluación Docente de la Universidad de Granada; 2007.

4. Herrera-Torres L, Cabo-Hernández JM. Experiencias piloto de implantación del sistema europeo de transferencia y acumulación de créditos ECTS. Reflexiones derivadas de su aplicación práctica en diferentes universidades españolas. Granada: Colección Educación Superior Europea, Comares; 2008 .

5. Herrera L, Lorenzo O, Rodríguez C. Las tutorías en el Espacio Europeo de Educación Superior: valoración de su implementación en la titulación de Maestro. Rev de Inv Psicoed. 2008; 6(1): 65-85.

6. Pimienta J. Constructivismo: estrategias para aprender a aprender. México: Pearson; 2004.

7. Lizzio A, Wilson K, Simons R. University Students' Perceptions of the Learning Environment and Academic Outcomes: implications for theory and practice. Stud. High. Educ. 2002; 27(1): 27-52. https://doi.org/10.1080/03075070120099359

8. Weinstein CE, Husman J, Dierking DR. Self-regulation interventions with a focus on learning strategies. En: Boekaerst M, Pintrich Pr, Zeidner M. Handbook of Self-Regulation. San Diego: Academic Press; 2000. p. 727-747.

9. Román-Sánchez JM, Gallego-Rico S. ACRA Escalas de estrategias de aprendizaje. Manual. Madrid: TEA; 1994.

10. Weinstein CE. LASSI User's Manual for those administering the Learning and Study Strategies Inventory (). $2^{\text {nd }}$ ed. Florida: H\&H Publishing Company, Inc.; 2002.

11. Bentham S. Psychology and Education (Routledge Modular Psychology).United Kingdom: Routledge; 2002.

12. Piñeiro I, Valle A, González-Cabanach R, Rodríguez-Martínez S. Autorregulación del aprendizaje y estrategias de estudio. En: Soler-Vázquez E, Núñez-Pérez JC, González-Pienda García JA, ÁlvarezPérez L. (Eds.), Estrategias de aprendizaje: concepto, evaluación e intervención. Madrid: Pirámide; 2002. p.17-38.

13. Allgood W, Risko V, Álvarez M, Fairbanks M. Factors that influence study. En: FFlippo RF, Caverly DC. Handbook of college reading and study strategies research. New Jersey: Erlbaum Publishers; 2000. 
p. 201-219.

14. Pintrich PR. A conceptual framework for assessing motivation and self-regulated learning in college students. Educ Psychol Rev. 2004; 16(4): 385-407. [Consultado agosto 31 2017] Disponible en: https://bit.ly/2AD4NR6

15. Valle-Arias A, González-Cabanach R, Nuñez-Pérez JC, Suárez-Riveiro JM, Piñeiro-Aguín I, RodríguezMartínez S. Enfoques de aprendizaje en estudiantes universitarios. Psicothema. 2000; 12(3): 368 75. [Consultado septiembre 3 2015]. Disponible en: https://bit.ly/2TKyjeV

16. Castelló M, Monereo C. El conocimiento estratégico en la toma de apuntes: un estudio en la educación superior. Infanc. aprendiz. 1999; 22(8): 25-42. https://doi.org/10.1174/021037099760246590

17. Nist SL, Simpson ML. College Studying. En: Kamil ML, Mosenthal PB, Pearson PD, Barr R. Handbook of Reading Research Volume III. New Jersey: Lawrence Erlbaum Associates. 2000. p. 645-66.

18. Ministerio de Planificación.Trayectoria de la pobreza y la distribución del Ingreso, período 1990 2009. Chile: MEDIPLAN 2009. [Consultado septiembre 27 2015]; Disponible en: https://bit.ly/2Cqz4Tt

19. Lemaitre M. Información y Educación Superior; un asunto de confianza. Santiago de Chile: Andros; 2003.

20. Tuckman B.The effect of learning and motivation strategies training on college students'achievement. J Coll Stud Dev. 2003; 44(3):430-37. https://doi.org/10.1353/csd.2003.0034

21. Gallardo G, Reyes P. Relación profesor-alumno: arista fundamental para el aprendizaje. Rev. Calidad de la Educación. 2010; 32:78-108. [Consultado septiembre 22 2015]. Disponible en: https://bit.ly/2QZv1Tq

22. Ministerio de Educación (MINEDUC). Síntesis de los principales enfoques, métodos y estrategias para la superación de la pobreza. Santiago: MIDEPLAN; 2002.

23. Cano F, Justicia FP. Estrategias de aprendizaje, especialidad y rendimiento Académico. Rev de psicología de la Educ.1991;(7): 69-86.

24. Bernad J. Modelo Cognitivo de Evaluación Educativa: Escalas de estrategias de Aprendizaje Contextualizado (ESEAC). Madrid: Narcea; 2000.

25. Gargallo B, Suarez JM, Pérez C. El Cuestionario CVEAPEU. Un instrumento para la evaluación de las estrategias de Aprendizajes de los estudiantes universitarios Rev. Relieve. 2009; 15(2);1-31.

26. Martín-Cabrera E, García-García LA, Torbay-Betancor A, Rodríguez-Blanco T. Estructura factorial y fiabilidad de un cuestionario de estrategias de aprendizaje en universitarios: CEA-U. Rev. Manuales de Psicología. 2007. [Consultado septiembre 29 2015]; 23:1-6. Disponible en: https://bit.ly/2Hj0twh

27. Camarero-Suárez F, Buey FM del, Herrero-Díez J. Estilos y estrategias de aprendizaje en estudiantes universitarios. Psicothema 2000; 12(4): 615-22. [Consultado octubre 15 2015]. Disponible en: https://bit.ly/2fuzPCh

28. Grimes SK. Targeting academic programs to student diversity utilizing learning styles and learningstudy strategies. J. Coll. Stud. Dev. 1995; 36(5):422-30.

29. Justicia F, Cano F. Concepto y medida de las estrategias y estilos de aprendizaje. En: Monereo C. (Coord). Las estrategias de aprendizaje. Barcelona: Doménech; 1997.

30. Sepúlveda-Carreño MJ, López-Quiero M, Torres-Vergara P, Luengo-Contreras J, Contreras-Muñoz E. Diferencias de género en el rendimiento académico y en el perfil de estilos y de estrategias de aprendizaje en estudiantes de química y farmacia de la Universidad de Concepción. Rev. estilos aprendiz, 2011; 7(7):135-50. [Consultado octubre 15 2015]. Disponible en: https: / / bit.ly/2RxdIy0

31. Fernández-González OM, Martínez-Conde Beluzan M, Mellipillán-Araneda, R. Estrategias de aprendizaje y autoestima: su relación con la permanencia y deserción universitaria. Estud Pedagóg, 2011;35(1):27-45. [Consultado junio 26 2017].Disponible en: https://bit.ly/10NQ5lB 
32. Kohler J. Relación entre habilidades intelectuales, estrategias de aprendizaje y rendimiento académico en estudiantes de psicología del 1 ro al 4to. Ciclo de una universidad particular. [Tesis]. Lima: Escuela Profesional de Psicología. Universidad de San Martín de Porres. 2008.

33. Cerioni M, Velez de Olmos G. Universidad Nacional de Rio Cuarto. Las Estrategias cognoscitivas de control y retención: Intervenciones instructivas. Argentina: Universidad del Río Cuarto, 1998. [Consultado octubre 26 2015]. Disponible en: https://bit.ly/2DhQZgR

34. Donolo D, Chiecher A, Rinaudo M, Estudiantes, estrategias y contextos de aprendizaje presenciales y virtuales. En: Primer Congreso virtual latinoamericano de educación a distancia, abril 2004. [Consultado noviembre 5 2015]. Disponible en: https://bit.ly/2FHJCAz

35. Castellanos-Cano S, Martín-Palacio ME, Cuesta-Izquierdo M, García-Cueto E. Cuestionario de Evaluación del Procesamiento Estratégico de la Información para Universitarios (CPEI-U). REMA. 2011; 16(2): 15-28. [Consultado noviembre 18 2015]; Disponible en: https://bit.ly/2ROB8OR

36. Vicerrectoría Académica. Universidad Santo Tomás. Plan Estratégico Institucional 2015-2018. Chile: Universidad Santo Tomas; 2016. p. 5 [Consultado noviembre 18 2015]. Disponible en: https://bit.ly/2BcK7x1

37. Beas J, Manterola M, Santa Cruz J, Carranza G. Capacitar monitores para enseñar a pensar: problemas y desafíos. En:VV. AA. Tercer encuentro Nacional sobre Enfoques Cognitivos Actuales en Educación. Santiago de Chile: Pontificia Universidad Católica de Chile; 1997. p. 57-78.

38. Alvarado-Martínez H, Sánchez-Soto I, Uribe-Santibañez M. Correspondencia entre estrategias de aprendizaje y rendimiento académico en estudiantes universitarios, Bol. invest. educ. 2000; 15: 7088. [Consultado noviembre 18 2015]. Disponible en: https://bit.ly/2RvmYml

39. Romo-Aliste ME, López-Real DG, Tovar-Martínez J, López-Bravo I. Estilos de aprendizaje de estudiantes de nutrición y dietética, Facultad de Medicina, Universidad de Chile, Rev. Praxis 2004; 5:9-24. [Consultado noviembre 27 2015]; Disponible en: https://bit.ly/2ssFm0e

40. Palella Stracuzzi S, Martins Pestana F. Metodología de la investigación cuantitativa. $3^{a}$. ed. Caracas: Fedupel; 2012.

41. Hernández R, Fernández C, Baptista MP. Metodología de la investigación. 5ª. ed. México: Mc Graw Hill; 2010.

42. Casal J, Mateu E. Tipos de Muestreo. Rev. Epidem. Prev. Med. 2003; 1(1), 3-7.

43. De la Fuente J, Justicia F. Escalas de estrategias de aprendizaje ACRA-Abreviada para alumnos universitarios. Revista Electrónica de Investigación Psicoeducativa y Psicopedagógica. 2003; 1(2): 1696-2095. [Consultado noviembre 30 2015]; Disponible en: https://bit.ly/2iNdOw0

44. Kaiser HF. An index of factorial simplicity. Psychometrika. 1974. 39: 31-36.

45. Catena-Martínez A, Ramos-Álvarez MM, Trujillo-Mendoza HM. Análisis Multivariado: un manual para investigadores. Madrid: Biblioteca Nueva; 2003.

46. Pérez E, Medrano L. Análisis factorial exploratorio: bases conceptuales y metodológicas Rev. argent. cienc. comport. 2010; 2(1): 58-66 [Consultado diciembre 09 2015]. Disponible en: https://bit.ly/2RCJDgr

47. Lolas F, Quezada A. Pautas éticas de investigación en sujetos humanos: nuevas perspectivas. Santiago de Chile: Programa Regional de Bioética OPS/OMS; 2003. 